\title{
Failure Investigation of A 4340 Steel Forge Knock-Out
}

\section{He*}

*Scot Forge Company, 8001 Winn Road, Spring Grove, IL 60081-0008

A 6" diameter 4340 forge knock-out (termed knock-out \#3) fractured into two halves during ID snapping of a nickel-based ring forging preform (Fig. 1). The knock-out below the fractured knockout (termed knock-out \#2), also made of 4340 steel, was buckled (non-uniform deformation) and cracked on the top face surface but did not split into multiple pieces (Fig. 2). This incident prompted an metallurgical investigation to determine if there are any abnormalities with the knock-out base material.

Knock-out \#3 fractured in compressive shear (Mode II fracture). A metallographic examination showed that its initiation site was heavily smeared by the metal flow during fracture that little useful evidence can be obtained (Fig. 3). Multiple adiabatic shear bands found near the fracture surface indicated the violent nature of this event (Fig. 4). Other than the cold worked region from the deformation occurred during the failure, the base microstructure is consistent with a 4340 steel in the quenched and tempered condition.

Knock-out \#2's crack on its top face surface was opened with an origin identified at the face/OD corner. Its cross section revealed evidence of cold work which was not unexpected given the event history (Fig. 5). Interestingly, along its tapered top surface, adiabatic shear bands were found, suggesting it also deformed rather rapidly (Fig. 6). Results of an axial tensile test survey along various locations on this knockout were consistent with the 363 HBW surface hardness obtained.

The grain sizes of both knock-outs were revealed using saturated aqueous picric acid with $\mathrm{HCl}$ and a wetting agent (Stepan $\mathrm{Nacconol}^{\circledR} 90 \mathrm{G}$ ). Knock-out \#3 appeared to have a slightly coarse grain size of ASTM No. 5-6 while knock-out \#2 had a more typical ASTM No. 7-8. The exact reason for the coarser grain size in knock-out \#3 is unknown but it was not considered the major reason for the failure. A chemistry check on both knock-outs showed good conformance to 4340 chemistry.

In conclusion, there were no metallurgical defects found on the knock-outs' base material. This type of knock-out had worked well for many years on most other ring forging snap operations. It is likely that a combination of factors contributed to the failure, they included,

1. the relative high aspect ratio (ID size vs. height) of the preform and the nickel superalloy forging's high flow stress resulted in very high pressure on the knock-outs;

2. Potential uneven preheat on knock-out \#2 caused it to buckle, creating an unstable loading position for knock-out \#3;

3. The knock-outs had uneven internal oxidation penetration and shallow surface cracks typical for the as-forged/heat treated surface, they may have lowered the overall crack resistance.

Several remedies were proposed to minimize the risk factor associated with this type of failures:

1. Only use knock-outs with machined surface to remove any surface stress concentrator.

2. For high flow stress grades like nickel based forgings, use a higher strength alloy grade for the knock-out, such as 300M; 
3. Do not preheat knock-outs;

4. Institute routine inspections of knock-outs and other forge tooling for signs of wear.

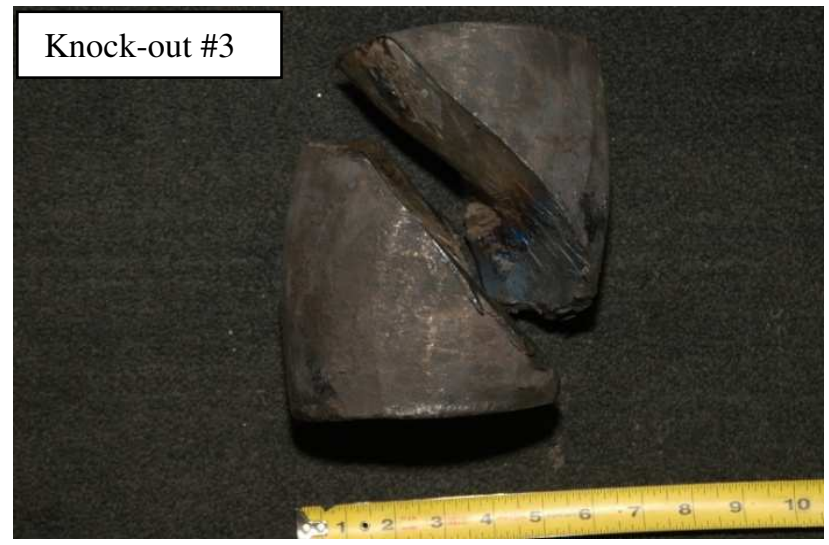

Fig. 1

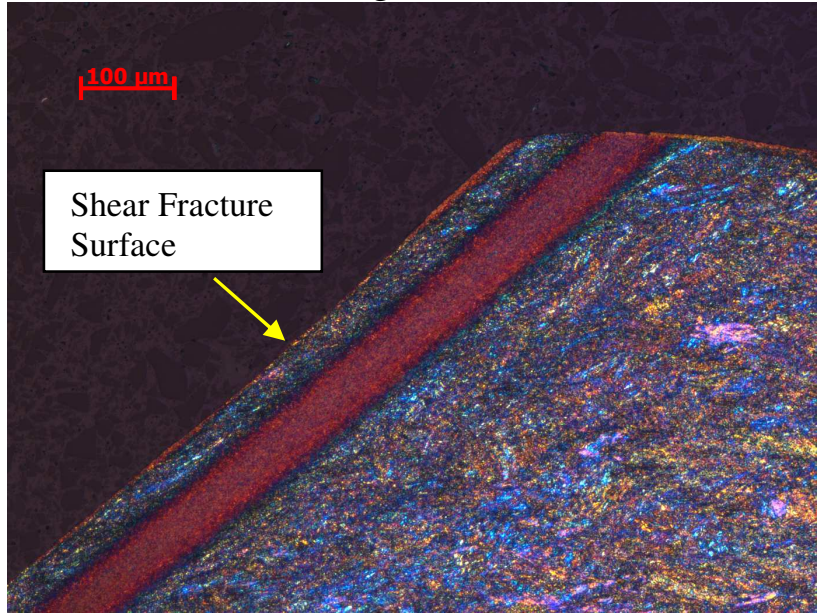

Fig. 3

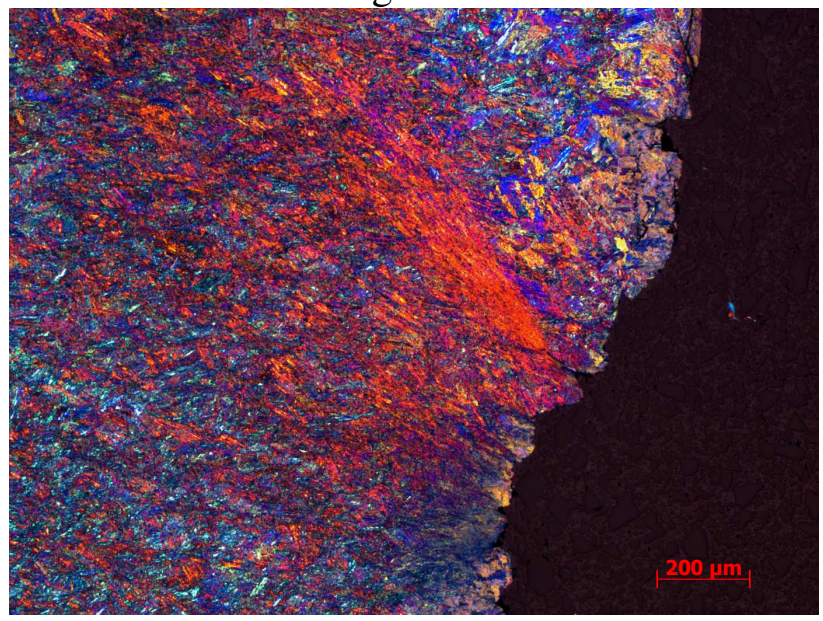

Fig. 5

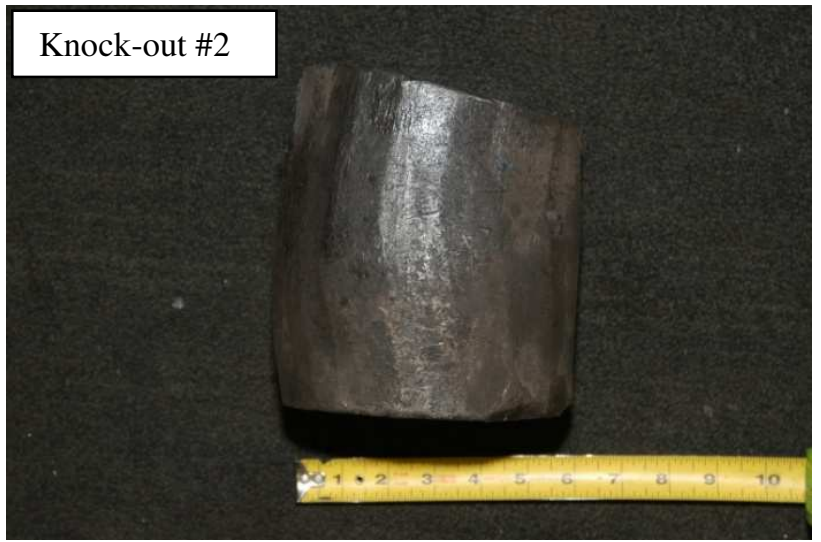

Fig. 2

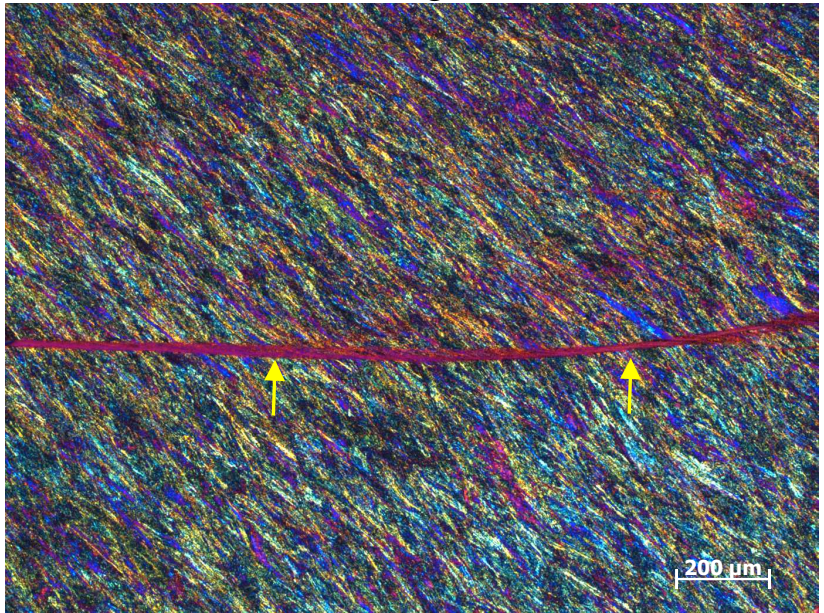

Fig. 4

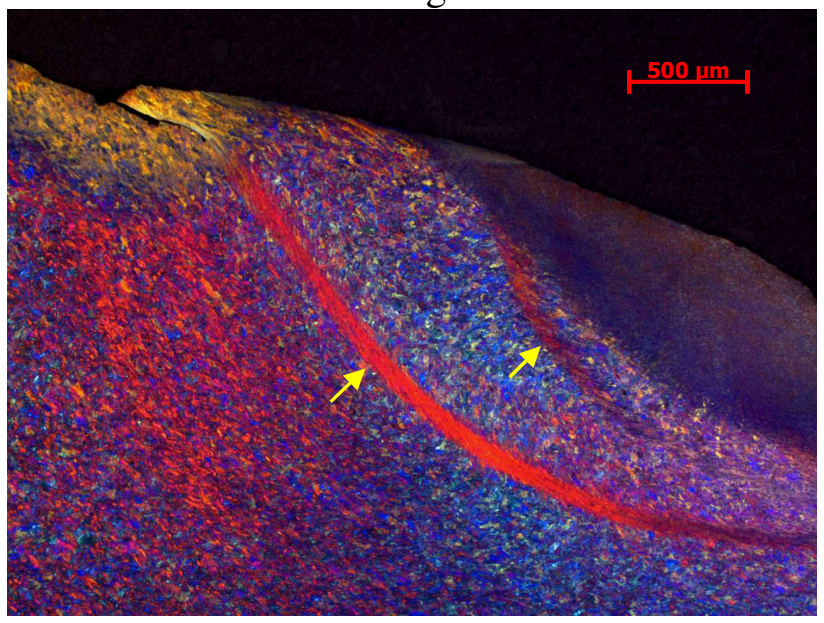

Fig. 6 